\title{
An oscillation effect during sequential alternations of unilateral amygdaloid stimulations within the kindling paradigm
}

\author{
JOHN GAITO \\ York University, 4700 Keele Street, Downsview M3J 1P3, Ontario, Canada
}

\begin{abstract}
In two experiments, rats were subjected to a sequence of electrical stimulations alternating from one amygdala to the contralateral one. Each phase of stimulation was for six convulsions prior to alternation to the other side. An oscillation effect resulted, involving low trials to six clonic convulsions and low latency to convulse for stimulation of one side, but high values of these measures for the contralateral site. The oscillation persisted, especially for the latency measure, even when one phase of bilateral stimulation preceded unilateral stimulation, when a 17- to 23-day rest period was inserted following a sequence of alternations and when two phases of bilateral stimulation occurred following postrest unilateral stimulations. The oscillation effect was less prominent in the number of trials to six convulsions data and almost nonexistent in duration of convulsion. Of 16 rats used in 15 to 19 alternating phases, 7 oscillated throughout all of these phases in latency data, but none showed oscillation over all phases in the other dependent variables.
\end{abstract}

If specific brain sites of an animal are stimulated unilaterally by low-intensity electrical current at periodic intervals, the animal's behavior changes in an orderly fashion from no response, to automatic behaviors (e.g., chewing, eye closure), and finally to clonic convulsions (CC). This event has been called the kindling effect (Goddard, McIntyre, \& Leech, 1969).

If one stimulates the amygdala (primary site) to a criterion (e.g., six CC), using $24 \mathrm{~h}$ as the intertrial interval, and then kindles the contralateral amygdala (secondary site), the criterion is reached within a fewer number of trials, but increased latency to convulsion results. Thus, both positive and negative transfer occurs (Goddard, McIntyre, \& Leech, 1969; McIntyre \& Goddard, 1973). If the animal is stimulated again at the primary site, it does not convulse on the first trial. A few trials are required to reach the $\mathrm{CC}$ state, thus indicating negative transfer.

An interesting question is: At what point in a sequence of alternations will a rat's behavior become stable, i.e., show no positive or negative transfer effects? In this paper, experiments are described in which 15 to 19 sequential alternations were provided with three trials per day. Rats tend to show an oscillation effect during these sequential alternations, especially in latency (i.e., time between onset of stimulation and onset of convulsion); they show low values with stimulation of the primary site and high values when the secondary site is being stimulated.

Research reported in this paper was supported by the President's NRC Fund (Grant 32) from York University and a grant from the Ontario Society for Crippled Children. I wish to acknowledge that Olive and Stephen Gaito constructed bipolar electrodes and that Paul Sanberg implanted these electrodes in the rats and prepared the brairs lices for histological analyses.
Some rats persist in this oscillation throughout the entire sequence of alternations.

\section{METHODS AND RESULTS}

Ten Wistar rats (approximately 150 days of age) were implanted bilaterally with bipolar electrodes in Experiment 1 . The electrodes consisted of nichrome wire with trimel coating, $0.127 \mathrm{~mm}$ in diameter, dipped once in Epoxylite. The brain coordinates for electrode implantation were the same as in many experiments in our laboratory (e.g., Gaito \& Gaito, 1974): $0.5 \mathrm{~mm}$ posterior to bregma, $4.5 \mathrm{~mm}$ from midline, $8.5 \mathrm{~mm}$ from skull.

Each rat was stimulated for $30 \mathrm{sec}$ with a $60-\mathrm{Hz}$ sine wave of $100 \mu \mathrm{A}$ (peak to peak), using a Lafayette stimulator, until six convulsions resulted. There were three trials per day, with $1 \mathrm{~h}$ between trials. On the day after the sixth convulsion, stimulation began at the secondary site and continued until six convulsions occurred. Then stimulation again at the primary site was instituted, etc. The rats went through 6 to 11 of these alternation phases. These rats constituted Group U.

A second experiment was conducted, parallel to the first one, with 10 rats, littermates of those used in Experiment 1 . These rats were stimulated bilaterally to a criterion of six convulsions first, and then unilateral stimulation phases were instituted (Group B).

In each experiment, for unilateral stimulation, five rats had the right amygdala as the primary site, and for five the left amygdala was the primary site.

As these experiments progressed, it was observed that most of the rats were oscillating from low values with stimulation of the primary site to high values with the secondary site, especially in latency (which was determined to the nearest second with a stopwatch). Rats were stopped at an alternation point showing low values (primary site for most rats), rested for a period of 17 to 23 days (individual rats reached the low point at different times), and then stimulated for four alternation phases beginning with the side previously showing high values (secondary site for most rats). After the fourth alternation phase (with most rats at the lowvalue point), all rats were stimulated bilaterally for two phases of six convulsions each. Finally, unilateral stimulation was begun again with the side previously indicating high values and continued through four alternating phases.

One rat from each group was discarded after the second phase of the first series of unilateral stimulation because over 40 trials 
Table 1

Means for Unilateral (U) and Bilateral (B) Groups Over Unilateral Stimulation Alternation Phases

\begin{tabular}{|c|c|c|c|c|c|c|c|c|c|c|c|c|c|}
\hline & \multicolumn{11}{|c|}{ Alternation Phases } & \multirow{2}{*}{$\begin{array}{c}\text { Primary } \\
\text { Site }\end{array}$} & \multirow{2}{*}{$\begin{array}{c}\text { Second- } \\
\text { ary } \\
\text { Site } \\
\end{array}$} \\
\hline & 1 & 2 & 3 & 4 & 5 & 6 & 7 & 8 & 9 & 10 & 11 & & \\
\hline & \multicolumn{13}{|c|}{ Latency of Convulsion (in seconds) } \\
\hline $\mathrm{U}$ & 9.0 & 12.6 & 7.9 & 11.5 & 9.3 & 12.8 & 10.0 & 11.2 & 7.7 & \multirow[t]{2}{*}{8.1} & \multirow[t]{2}{*}{3.6} & 8.4 & 11.8 \\
\hline B & 8.1 & 12.8 & 9.9 & 13.3 & 9.9 & 14.1 & 7.7 & 10.5 & 3.7 & & & 8.8 & 13.3 \\
\hline & \multicolumn{13}{|c|}{ Number of Trials to Six Convulsions } \\
\hline $\mathbf{U}$ & 17.3 & 21.6 & 6.8 & 10.1 & $7.3^{1}$ & 8.1 & 8.6 & 9.1 & 7.0 & \multirow[t]{2}{*}{7.0} & \multirow{2}{*}{6.0} & 6.8 & 12.0 \\
\hline B & 9.8 & 14.8 & 10.1 & 10.1 & 8.7 & 7.0 & 7.4 & 7.0 & 6.0 & & & 9.0 & 10.6 \\
\hline & \multicolumn{13}{|c|}{ Duration of Convulsion (in seconds) } \\
\hline $\mathrm{U}$ & 23.8 & 27.6 & 29.0 & 29.9 & 28.8 & 31.4 & 28.9 & 30.3 & 24.9 & \multirow[t]{2}{*}{26.2} & \multirow[t]{2}{*}{23.6} & 27.9 & 29.5 \\
\hline B & 27.0 & 30.1 & 29.6 & 31.9 & 29.8 & 29.1 & 23.7 & 27.0 & 26.8 & & & 27.7 & 30.3 \\
\hline
\end{tabular}

were required to reach criterion. From results of previous work (Gaito \& Gaito, 1974), it was apparent that one of the electrodes was not in the amygdala. At the completion of the experiments, histological analyses using a photographic method (Hutchinson \& Renfrew, 1967) were conducted on all rats except six oscillators which were set aside for later research. These analyses confirmed that the electrode tips were in both amygdalae for all rats except these two. In one rat, one electrode was in the optic 'tract; in the other, an electrode was at the intersection of the globus pallidus and caudate putamen.

The results were clear-cut, although unexpected and somewhat puzzling. Most rats both in the group stimulated bilaterally first (Group B) and in the unilateral group (Group U) showed an oscillation effect, varying from low values with stimulation of the primary site to high values with the secondary site. The oscillation was most pronounced in the latency data (Table 1). Fourteen of 18 rats showed the oscillation over most or all alternation phases. This oscillation effect involved unidirectional negative transfer, from primary to secondary site.

There was a similar tendency to oscillate in the number of trials to six convulsions data as well (Table 1). However, in spite of the fact that 14 of 18 rats showed moderate to good oscillation, the effect was not as prominent as in the latency data because it tended to dissipate earlier for a few rats. Furthermore, there were three rats which missed one phase of oscillation and began to oscillate in the opposite direction. This latter aspect would obscure the oscillation tendency in group data.
In the duration of convulsion data, there was some tendency towards oscillation (Table 1). However, only 6 of 18 rats oscillated in this measure.

The mean of the primary site was lower than that for the secondary site in each of the dependent variables for both the unilateral and bilateral groups (Table 1). The difference was least in the duration measure.

Several rats oscillated in each of the three dependent variables, over all or most of the phases. Table 2 shows typical data for one such rat from the unilateral group and one from the bilateral group.

The rest interval tended to disrupt the oscillation effect in one dependent variable (number of trials to six convulsions, Table 3 ). On the first phase (which was an expected high-value phase and a secondary site for most rats), most of the rats achieved the criterion of six convulsions in six trials (13 of 16 rats ${ }^{1}$ ). When this value for these 13 rats was compared to that for the next to last prerest phase (which involved stimulation of the high side also), 11 of the 13 showed a decrease in value, with a value comparable to that attained on the low side in the last prerest phase. The other two rats had equal values in the two phases. The three rats which did not attain criterion in six trials reverted to values achieved during the early phases of the prerest phases $(14,17$, and 18 trials to criterion). These three values tend to inflate the mean value of 7.9 shown in Table 3 for

Table 2

Typical Oscillation Data (Means)

\begin{tabular}{|c|c|c|c|c|c|c|c|c|c|c|c|}
\hline & \multicolumn{11}{|c|}{ Alternation Phases } \\
\hline & 1 & 2 & 3 & 4 & 5 & 6 & 7 & 8 & 9 & 10 & 11 \\
\hline & \multicolumn{11}{|c|}{ Rat No. 18 (Unilateral) } \\
\hline Latency & 6.2 & 12.0 & 3.3 & 8.7 & 4.8 & 10.3 & 5.8 & 8.2 & 4.2 & 5.2 & 1.8 \\
\hline Number of Trials & 7 & 14 & 7 & 11 & 6 & 11 & 6 & 10 & 6 & 8 & 6 \\
\hline \multirow[t]{2}{*}{ Duration } & 24.8 & 35.2 & 33.5 & 39.3 & 27.7 & 32.7 & 25.8 & 27.8 & 28.3 & 21.7 & 28.2 \\
\hline & \multicolumn{11}{|c|}{ Rat Number 15 (Bilateral) } \\
\hline Latency & 5.3 & 11.2 & 5.8 & 10.7 & 8.5 & 10.3 & 8.8 & & & & \\
\hline Number of Trials & 6 & 20 & 7 & 11 & 8 & 9 & 8 & & & & \\
\hline Duration & 29.3 & 33.7 & 31.5 & 34.3 & 37.3 & 33.3 & 36.8 & & & & \\
\hline
\end{tabular}


Table 3

Postrest Data

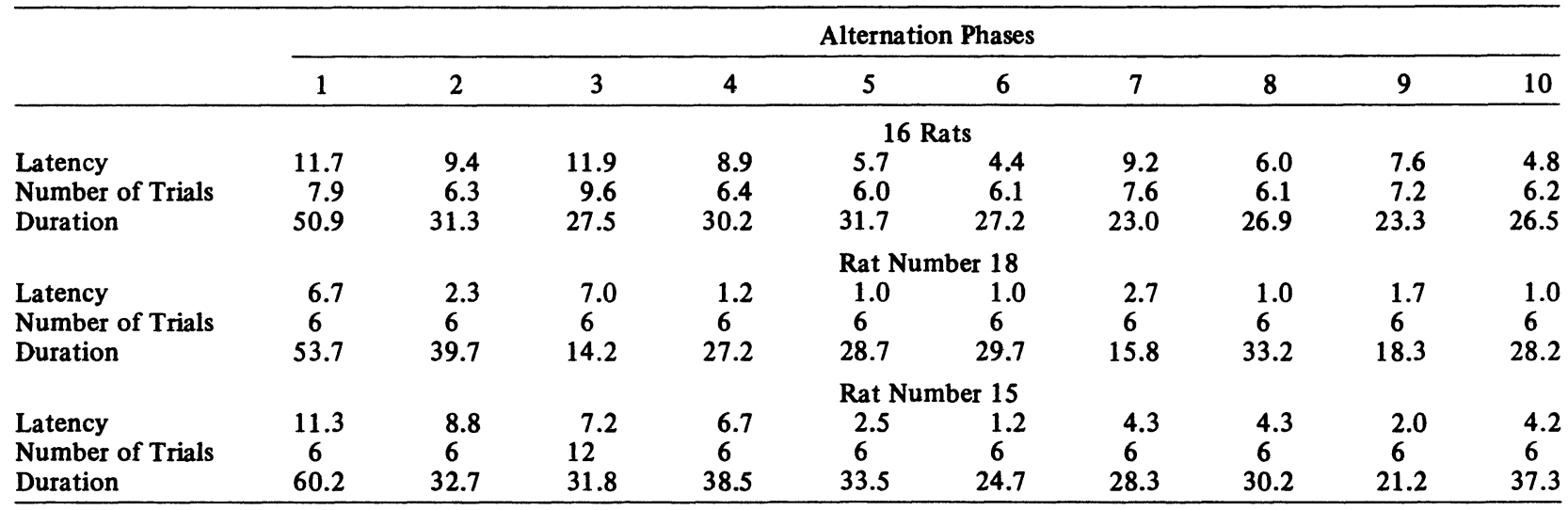

Note-Phases 1.4 and 7.10 are unilateral stimulation sequences; 5 and 6 are bilateral phases. Alternation Phase 1 is for the side contralateral to last stimulation site prior to the rest interval; Alternation Phase 7 is for side contralateral to side stimulated in Phase 4. Phases 1 and 7 involve stimulation of the secondary site for most rats, 13 of 16.

the first postrest phase. If the median had been used instead of the mean for this situation, the median would have been 6 , a more representative value in this one case. Thus, the rest period initially appeared to bring about an interruption of oscillation for most rats, but increased the negative transfer effect drastically for three rats.

By the third postrest phase, most rats were again showing high values; only 2 of the 16 rats achieved the criterion in six trials. The second and fourth phases provided low values (most rats required only six trials to achieve criterion). Thus, although the rest period allowed the negative transfer effect to dissipate during the first phase for most rats, this effect was prominent again by the third phase.

In the latency data, where the oscillation effect was most pronounced, the rest interval had no effect for most rats (Table 3). Most animals had high values on the first and third phases, and low values on the second and fourth ones.

As in the prerest trials, oscillation tended not to occur in the duration data. However, the mean of the first phase was greater in all rats on the first postrest phase than for the second phase, a difference of approximately $20 \mathrm{sec}$.

The two phases of bilateral stimulation produced similar results, as had the rest interval. The oscilla- tion effect was pronounced in latency data, but of much less magnitude in the trials to criterion data. In the latter dependent variable, the number of rats reaching the criterion in six trials was: Phase 7,8 ; Phase 8, 14; Phase 9, 12; Phase 10, 14; the bilateral stimulation disrupted the oscillation effect partially. The means on the two bilateral phases for these two dependent variables was lower than, or as low as, those on any previous phase for every rat.

The mean values for two rats in Table 3 show clearly the strong oscillation effect in latency data in one rat but only partially in the other, and the weakness of the criterion measure in indicating this effect.

Of the 16 rats which were involved in both prerest and postrest sequences, seven persisted in oscillating over all 15 to 19 phases in latency data, in spite of the insertion of the rest interval and bilateral stimulation phases (Table 4). No rats oscillated over all sequences in the other dependent variables.

Stimulation need not be in the amygdala to achieve oscillation. One of the two rats with misplaced electrodes (in the caudate putamen-globus pallidus area) was run for three alternation phases beyond the first unilateral stimulation of the primary site. This rat oscillated on the three phases after the first one in criterion data. Even though 50 trials were required

Table 4

Consistent Oscillators in Latency Measure (Means)

\begin{tabular}{|c|c|c|c|c|c|c|c|c|c|c|c|c|c|c|c|c|c|c|c|c|c|}
\hline \multirow{2}{*}{$\begin{array}{c}\text { Rat } \\
\text { Number } \\
\end{array}$} & \multicolumn{11}{|c|}{ Prerest Phases } & \multicolumn{10}{|c|}{ Postrest Phases } \\
\hline & 1 & 2 & 3 & 4 & 5 & 6 & 7 & 8 & 9 & 10 & 11 & 1 & 2 & 3 & 4 & 5 & 6 & 7 & 8 & 9 & 10 \\
\hline 1 & 9.2 & 15.3 & 7.0 & 16.7 & 9.2 & 18.0 & 7.0 & & & & & 11.5 & 10.0 & 16.8 & 7.0 & 1.3 & 1.0 & 9.7 & 2.0 & 9.2 & 3.0 \\
\hline 2 & 12.2 & 13.8 & 5.0 & 15.2 & 5.3 & 15.8 & 6.5 & 14.2 & 5.2 & 14.3 & 2.5 & 11.2 & 5.0 & 9.7 & 3.5 & 1.5 & 1.0 & 6.5 & 1.0 & 6.0 & 1.0 \\
\hline 12 & 5.3 & 14.5 & 6.0 & 11.3 & 4.2 & 13.5 & 7.3 & & & & & 12.2 & 4.3 & 8.8 & 5.5 & 3.7 & 2.2 & 9.5 & 4.3 & 12.8 & 3.8 \\
\hline 13 & 4.5 & 5.8 & 6.8 & 13.5 & 4.5 & 14.0 & 4.7 & & & & & 15.2 & 5.0 & 8.8 & 5.2 & 1.8 & 2.0 & 7.5 & 1.7 & 9.3 & 1.8 \\
\hline 17 & 6.2 & 11.8 & 9.0 & 11.3 & 9.7 & 14.0 & 7.7 & 10.5 & 3.7 & & & 7.5 & 5.2 & 14.2 & 4.3 & 2.0 & 1.0 & 7.2 & 3.2 & 4.5 & 1.0 \\
\hline 18 & 6.2 & 12.0 & 3.3 & 8.7 & 4.8 & 10.3 & 5.8 & 8.2 & 4.2 & 5.2 & 1.8 & 6.7 & 2.3 & 7.0 & 1.2 & 1.0 & 1.0 & 2.7 & 1.0 & 1.7 & 1.0 \\
\hline 19 & 8.8 & 19.5 & 13.0 & 17.7 & 13.8 & 19.0 & 11.5 & & & & & 18.2 & 11.0 & 15.7 & 10.8 & 6.8 & 8.8 & 14.0 & 9.2 & 10.3 & 8.0 \\
\hline
\end{tabular}


to reach criterion during stimulation of the primary site on the first phase, latencies were lower on this phase and on the third phase, showing excellent oscillation for the four phases.

Fourteen of the 18 rats used in the data analyses showed the oscillation effect (primary oscillation). If the one with a misplaced electrode is included also, 15 of 19 rats achieved oscillation. Three other rats showed secondary oscillation (low values in secondary site, high values in primary site) before rest and continued this oscillation in some postrest phases. Therefore, 18 of 19 rats demonstrated oscillation, either primary or secondary on all or some portion of the 15 to 19 phases.

\section{DISCUSSION}

These results seem to indicate that oscillation is a unique phenomenon peculiar to unilateral stimulation. One might suggest that the oscillation observed in these two experiments is a chance effect. But the likelihood of a number of rats showing a systematic change from low to high (or high to low) on successive phases is extremely low. Also, one might state that oscillation is based on poor placement of one of the two electrodes. Although this latter explanation sounds like a reasonable one, there are several observations which make it appear unlikely.

(1) If poor placement is the basis for oscillation, the number of primary oscillators should be approximately equal to the number of secondary oscillators. However, there tend to be more primary oscillators than secondary oscillators; 15 of 18 rats showed primary oscillation.

(2) Although the latency and criterion measures go in the same direction in many cases (especially during the early phases), they are relatively independent on other occasions. Oscillation occurs more frequently in latency data than in the criterion measure. Poor electrode placement would be expected to affect the criterion measure, e.g., by requiring more trials to achieve the six CC in early phases. However, this effect need not be influencing the latency measure. In fact, the electrode does not have to be in the amygdala to achieve oscillation. One rat with a misplaced electrode (in the caudate putamen-globus pallidus area) provided excellent oscillation in latency data. Even though 50 trials were required to reach criterion during stimulation on the first phase, latencies were lower on this phase and on the third phase, showing excellent primary oscillation for the four phases on which this rat was run.

McIntyre and Goddard (1973) reported both positive and negative transfer effects when using sequen- tial alternation of stimulation over three phases with one trial per day. Our results with three trials a day do not show positive transfer in any measure, but do indicate clearly the persistence of negative transfer in one direction only over multiple phases in latency and criterion measures. Furthermore, our results indicate that a rest period of 17 to 23 days and bilateral stimulation has little effect on the oscillation effect in the latency measure but has a disruptive, or partially disruptive, effect in the criterion data.

In these experiments, the unidirectional negative transfer or inhibitory process appears to be responsible for the oscillation effect. The strength of the process would seem to vary with different stimulation conditions; possibly strength would increase with massing of trials, increasing number of convulsions (McIntyre \& Goddard, 1973), and a number of other factors. This inhibitory process may involve the same chemical which produced the interanimal retardation effect in the development of kindling in naive recipient rats (Gaito, 1976; Gaito \& Gaito, 1974).

This unilateral sequential alternation procedure appears to be useful for obtaining information on some aspects of underlying mechanisms via the oscillation effect. By determining variables which minimize or maximize this effect, one could gain clues concerning brain mechanisms which are responsible for the kindling effect.

\section{NOTE}

1. Of the 18 rats, only 16 were used in the postrest phase. These 16 had values in criterion or latency measures which were lower than those in the previous phase. One rat did not show a definite low point in the last prerest phase and was discarded; it was not an oscillator. The other animal lost the electrodes during the rest interval.

\section{REFERENCES}

Gaito, J. Pairing of the transfer experiment with the kindling paradigm: A summary of results. Bulletin of the Psychonomic Society, 1976, 7, 50-52.

Gaito, J., \& Gaito, S. T. Interanimal negative transfer of the kindling effect. Physiological Psychology, 1974, 2, 379-382.

Goddard, G. V., McIntyre, D. C., \& Leech, C. K. A permanent change in brain function resulting from daily electrical stimulation. Experimental Neurology, 1969, 25. 295-330.

Hutchinson, R. R., \& Renfrew, J. W. A simple histological technique for localizing electrode tracks and lesions within the brain. Journal of the Experimental Analysis of Behavior, 1967. 10, 277-280.

MCINTYRe, D. C., \& Goddard, G. V. Transter, interference and spontaneous recovery of convulsions kindled from the rat amygdala. Electroencephalography and Clinical Neurophysiologı, 1973, 35. 533-543.

(Received for publication Decemher 8, 1975; revision accepted April 1, 1976.) 